\title{
Les besoins en matière de soins de soutien parmi les proches des hommes atteints d'un cancer prostatique avancé
}

par Nancy Carter, Denise Bryant-Lukosius, Alba DiCenso, Jennifer Blythe et Alan J. Neville

\begin{abstract}
Abrégé
Cette étude exploratoire a dégagé les besoins en matière de soins de soutien des proches d'hommes atteints d'un cancer de la prostate hormono-sensible (HS) et d'un cancer de la prostate androgénoindépendant (AI). Dans le cadre de groupes de discussion et d'entrevues individuelles, nous avons demandé à huit membres de la famille d'hommes atteints d'un cancer HS et à 11 membres de la famille d'hommes atteints d'un cancer AI d'indiquer leurs besoins en matière de soins de soutien et de recommander des stratégies susceptibles d'améliorer les soins de façon à répondre à ces besoins. Les besoins non comblés signalés par les deux groupes comprenaient le manque d'information et l'incertitude par rapport à l'avenir. Les besoins non comblés particuliers au groupe AI concernaient le fardeau des soignants, l'assistance pratique et l'isolement. Cet article comprend les implications de la recherche concernant l'amélioration des services de soutien offerts aux familles touchées par le cancer prostatique avancé.
\end{abstract}

Le groupe le plus touché par le cancer de la prostate est celui des hommes de plus de 65 ans. Dans les cas de cancer de la prostate avancé ou métastatique (CPA), les problèmes courants, dont les atteintes à la fonction urinaire, la fatigue et la douleur, sont aggravés par d'autres enjeux de santé liés à l'âge et à la comorbidité (Harden, 2005). Il existe deux groupes de patients distincts qui vivent avec le CPA. Les hommes atteints d'un cancer de la prostate hormono-sensible (HS) reçoivent un traitement de privation androgénique, qui a un effet de féminisation, dont la dysfonction érectile. Tôt ou tard, la maladie de la plupart des patients finit par « résister » aux hormones, c'est-à-dire qu'elle devient androgéno-indépendante (AI), et on se tourne alors vers les traitements palliatifs accompagnés de chimiothérapie, de radiothérapie et de soins de soutien (McMurtry \& McMurtry, 2003). Comme toute maladie grave, le cancer de la prostate touche la famille au complet, en particulier les conjointes ou les partenaires (Maliski, Heilemann \& McCorkle, 2002). Le traitement du cancer de la prostate a des effets secondaires sur la sexualité qui nuisent aux relations intimes et accroissent le niveau de stress lié à l'adaptation à la maladie d'un proche.

Des études passées indiquent que les partenaires de vie des hommes ayant un cancer prostatique signalent souvent des niveaux de détresse plus élevés que les patients eux-mêmes (Couper et coll., 2006; Resendes \& McCorkle, 2006) et que la qualité de vie des partenaires diminue à mesure que les problèmes des patients s'aggravent (Kornblith, Herr, Ofman, Scher \& Holland, 1994). Les difficultés signalées par les partenaires comprennent la détresse liée à l'inquiétude et à les tensions liées au rôle (Harden, 2005), le manque d'information (Butler et coll., 2000), les difficultés liées à l'intimité et à la sexualité (Couper et coll., 2006; Crowe \& Costello, 2003; Sanders, Pedro, Bantum \& Galbraith, 2006) et les enjeux de communication avec le partenaire malade (Boehmer \& Clark, 2001b; Hawes et coll., 2006; Lavery \& Clarke, 1999). Le stade de la maladie est rarement cerné dans les études sur les partenaires d'hommes ayant le cancer de la prostate (Crowe \& Costello, 2003; Feltwell \& Rees, 2004; Harden, Northouse \& Mood, 2006; Manne, Babb, Pinover, Horwitz \& Ebbert, 2004; Rees et coll., 2003). Peu d'études ont été conçues pour comprendre les besoins des partenaires d'hommes vivant avec un CPA, et aucune étude ne fait de distinction entre les partenaires d'hommes atteints d'un cancer HS ou d'un cancer AI.

Cette étude fait partie d'un vaste programme de recherche sur le rôle des infirmières en pratique avancée dans la gestion du CPA. Les chercheuses ont utilisé un processus participatif, basé sur des données probantes et axé sur le patient pour le développement, la mise en œuvre et l'évaluation du rôle d'infirmière en pratique avancée. Ce cadre en neuf étapes, baptisé PEPPA par Bryant-Lukosius et DiCenso (2004), met l'accent sur l'importance de l'évaluation des besoins des patients et de leur famille et vise à cerner les problèmes et objectifs prioritaires et à déterminer quels services de santé additionnels sont nécessaires et qui devrait les offrir.

Dans cet article, nous ciblons les besoins des membres de la famille d'hommes vivant avec un CPA. Plus précisément, nous avons abordé les questions de recherche suivantes : 1) Quels sont les besoins en matière de soins de soutien parmi les proches d'hommes atteints d'un cancer de la prostate HS et AI et en quoi ces besoins diffèrent-ils entre les deux groupes?; 2) Quelles sont les stratégies recommandées pour l'amélioration des soins de soutien offerts aux membres de la famille afin que leurs besoins soient satisfaits?

\section{Au sujet des auteures}

Nancy Carter, inf., Ph.D., École des sciences infirmières, Faculté des sciences de la santé, Université McMaster, 1200 Main Street West, HSC-3N25A, Hamilton, ON L8N 3Z5. Tél : 905-525-9140 poste 22221; Courriel : carternm@mcmaster.ca

Denise Bryant-Lukosius, inf., Ph.D., Professeure agrégée, École des sciences infirmières et Département d'oncologie, Faculté des sciences de la santé, Université McMaster; également affectée au Centre de cancérologie Juravinski, Hamilton, ON.

Alba DiCenso, inf., Ph.D., Professeure, École des sciences infirmières et Département d'épidémiologie clinique et de biostatistique, Faculté des sciences de la santé, Université McMaster, Hamilton, ON.

Jennifer Blythe, Ph.D., Professeure agrégée, École des sciences infirmières, Faculté des sciences de la santé, Université McMaster, Hamilton, ON.

Alan J. Neville, MBChB, M.Ed., MRCP, FRCP(c), Professeur, Médecine, vice-doyen, Programme de médecine de premier cycle, Faculté des sciences de la santé, Université McMaster, Hamilton, ON. 


\section{Méthodes}

Cette étude était une évaluation des besoins. Nous avons employé la méthode descriptive qualitative décrite par Sandelowski (2000). Nous avons recueilli de l'information auprès des membres de la famille d'hommes vivant avec un cancer de la prostate HS et $\mathrm{AI}$ et ce, séparément pour chaque groupe. Cette séparation en deux groupes se doit à des différences au niveau des besoins de soins de santé cernés chez les hommes atteints de différentes formes de CPA (Bryant-Lukosius et coll., en instance de publication). Les proches admissibles comprenaient les épouses, les partenaires et les enfants des patients ayant un CPA. Nous avons recruté nos participantes dans différents milieux, dont des cabinets d'urologues, un centre de cancérologie et des groupes de soutien locaux pour hommes ayant un cancer prostatique. Bon nombre des participantes provenaient d'une étude parallèle sur les patients atteints d'un CPA (Carter, Bryant-Lukosius, DiCenso, Blythe \& Neville, 2009).

\section{Collecte et analyse des données}

Nous avons recueilli les données par le biais d'entrevues en profondeur et de groupes de discussion. Avant la collecte, les participantes ont rempli un formulaire de consentement et un bref questionnaire de nature démographique. Les participantes interviewées ont pris part à une entrevue semi-structurée d'une durée de 40 à 90 minutes, tandis que les membres des groupes de discussion ont participé à une séance de 60 à 90 minutes. La chercheuse principale (Nancy Carter) a mené toutes les entrevues et dirigé tous les groupes de discussion. Nous avons demandé aux participantes de décrire les effets du CPA sur leur vie et les problèmes auxquels elles faisaient face. Nous leur avons également demandé d'indiquer si leurs besoins étaient comblés et d'établir l'ordre de priorité des efforts futurs visant à améliorer les soins. Nous avons utilisé le guide d'entrevue semi-structurée pour les entrevues et les groupes de discussion (Annexe A). À la fin des entrevues et des groupes de discussion, la chercheuse a résumé les besoins prioritaires et les stratégies suggérées et a récapitulé les principaux thèmes afin de valider et clarifier l'information (vérification par les membres). Toutes les discussions, entrevues et réflexions ont fait l'objet d'un enregistrement sonore et ont été transcrites.

Nous avons analysé les données à mesure qu'elles étaient recueillies. Les membres de l'équipe de recherche ont lu les transcriptions initiales indépendamment et ont élaboré un schéma de codage. La chercheuse principale et deux assistantes de recherche ayant une expérience des soins infirmiers en oncologie ont examiné toutes les transcriptions et utilisé le schéma de codage et le logiciel N-Vivo pour élaborer les catégories principales. Nous avons regroupé les codes dans des catégories et les thèmes importants se sont dégagés. La chercheuse principale et ses assistantes ont discuté de la saturation des données et ont convenu du moment où aucun nouveau thème n'émergeait et où la collecte de données pouvait cesser. Le comité d'éthique de la recherche de l'université et le comité d'éthique de la recherche en soins hospitaliers ont approuvé la réalisation de cette étude.

\section{Résultats}

\section{Caractéristiques des participantes}

Nous avons recueilli des données auprès de 19 proches. Onze d'entre eux étaient des proches d'hommes atteints d'un cancer AI, et huit étaient des proches d'hommes ayant un cancer HS (voir le tableau). Dix des onze proches AI ont pris part à l'un des trois groupes de discussion. Parmi ces dix personnes, neuf étaient des épouses, et une était une fille qui a participé avec sa mère. Nous avons interviewé une proche AI chez elle en compagnie de son mari. L'âge des épouses d'hommes vivant avec un cancer AI oscillait entre
53 et 85 ans. La fille qui a participé avec sa mère avait 56 ans. Nous avons également interviewé huit membres de la famille d'hommes atteints d'un cancer HS (six épouses, un fils et une fille). L'âge des six épouses allait de 63 à 85 ans. Le fils avait 52 ans et sa mère n'était pas une des participantes à l'étude. La fille avait 32 ans et sa belle-mère était une participante.

\section{Les besoins des proches en matière de soins de soutien}

L'analyse des données a révélé cinq grands thèmes liés aux besoins en matière de soins de soutien. Malgré le fait que les deux groupes de proches (HS et AI) signalaient des enjeux et des expériences semblables par rapport au cancer de la prostate, le groupe AI mentionnait une détérioration notable du niveau de bien-être et un lourd fardeau lié à la maladie. Les deux groupes ont exprimé un besoin d'information et une incertitude par rapport à l'avenir. Certains régimes thérapeutiques, notamment la chimiothérapie, entraînaient un niveau plus élevé de besoins parmi le groupe AI. Les données sur ce groupe révélaient des enjeux liés au fardeau des soignants, au besoin d'assistance pratique et à l'isolement. Nous décrivons ces thèmes en plus grand détail ci-dessous.

Besoins similaires des proches des hommes

ayant un cancer de la prostate HS et de

ceux ayant un cancer de la prostate AI

Besoins en matière d'information

Les proches des groupes HS et AI souhaitaient tous recevoir plus d'information sur les traitements et les effets secondaires, sur les services de soins de soutien offerts et sur l'évolution de la maladie. Une participante du groupe AI a indiqué que : "Selon moi, le problème est le manque d'information. On ne nous dit rien sur ce qui est disponible. Donc, à moins de demander explicitement, nous ne savons rien. Parfois, nous ne savons pas quelle question poser, parce que nous ne savons pas ce qui existe. » Les participants étaient d'avis qu'ils ne recevaient pas suffisamment d'information et sentaient qu'ils devaient poser les questions appropriées pour obtenir les renseignements voulus. Les participantes aux groupes de discussion partageaient souvent de l'information. La plupart des épouses indiquaient que les médecins étaient leurs principales sources d'information, mais que les renseignements qu'elles recevaient lors des rendez-vous médicaux étaient soient excessifs, soit difficiles à comprendre. Plusieurs proches plus âgés ont signalé que leur perte d'acuité auditive rendait ces rendez-vous dans les cabinets des médecins encore plus frustrants. Ils ont également mentionné qu'ils se sentaient pressés lors de ces rencontres. Les femmes hésitaient à poser des questions lors des rendez-vous car elles ne savaient pas si c'était à elles de le faire.

Tableau 1. Description des proches ayant participé à l'étude

\begin{tabular}{|l|l|l|}
\hline & $\begin{array}{l}\text { Groupe } \\
\text { androgéno- } \\
\text { indépendant } \\
n=11\end{array}$ & $\begin{array}{l}\text { Groupe } \\
\text { hormono- } \\
\text { sensible } \\
n=8\end{array}$ \\
\hline Nombre d'épouses & 10 & 6 \\
\hline $\begin{array}{l}\text { Âge moyen des } \\
\text { épouses (étendue) }\end{array}$ & $\begin{array}{l}71 \text { ans } \\
(53-85 \text { ans })\end{array}$ & $\begin{array}{l}69 \text { ans } \\
(63-85 \text { ans })\end{array}$ \\
\hline Nombre d'enfants adultes & 1 & 2 \\
\hline Âge des enfants adultes & 56 ans & 32 ans, 52 ans \\
\hline $\begin{array}{l}\text { Collecte de données : } \\
\text { Groupes de discussion } \\
\text { Entrevues }\end{array}$ & 10 & 0 \\
\hline
\end{tabular}


Une participante du groupe HS s'est exprimée ainsi : « Mes questions sont différentes de celles de mon mari, mais je ne veux pas le bouleverser, vous comprenez. »Comme cette femme ne posait pas les questions qu'elle avait, ses besoins d'information n'étaient pas satisfaits.

Les proches plus jeunes trouvaient de l'information dans Internet, mais bon nombre des participantes plus âgées n'avaient pas recours à cette ressource. Un risque lié aux recherches sur Internet est l'excès d'information. Une des femmes a indiqué que « l'information est très puissante, mais il faut faire attention, parce que si on ne sait pas mettre même un seul renseignement en contexte, il peut être tout à fait terrifiant. Nous avons cessé de chercher sur Internet pour cette raison. »

\section{Incertitude par rapport à l'avenir}

Les participants des groupes HS et AI ont tous discuté de leurs sentiments d'incertitude par rapport à l'avenir et de leur frustration de ne pas savoir ce à quoi s'attendre à mesure que le cancer évoluait. Une participante du groupe HS a fait valoir que : "Essentiellement, il est exactement comme avant, sauf qu'il y a un compteur d'antigènes prostatiques spécifiques au-dessus de sa tête. On voudrait savoir quand le compteur va afficher une mesure différente. » La fragilité de la condition de son mari était une menace constante, malgré le fait qu'il semblait ne pas avoir changé depuis l'apparition de la maladie. Les membres de la famille se demandaient souvent de quelle façon la progression du cancer allait affecter leur être cher. Cette participante du groupe AI était d'avis que les fournisseurs de soins ne dévoilaient pas toute l'information : «Que nous réserve l'avenir? Personne n'a de boule de cristal ou personne ne nous le dit. Avec un peu de chance, nous pourrons continuer comme ça pendant quelques années. Je ne sais tout simplement pas. Mais je ne veux pas le voir vivre et souffrir. » Elle pouvait endurer la condition actuelle de son mari, mais elle craignait qu'il souffre à l'avenir. Les conjointes n'étaient pas certaines de pouvoir composer avec la détérioration de la condition de leur mari. Une participante du groupe HS a indiqué que : «Je ne sais pas ce qui se passerait s'il devenait soudainement malade. Il m'a dit un jour "N'essaie surtout pas de me garder à la maison", et j'ai répondu "Oui, je sais que je ne pourrais pas endurer ça. Je ne le pourrais tout simplement pas.” »

\section{Besoins particuliers des proches des hommes atteints d'un cancer de la prostate AI}

Outre les besoins indiqués ci-dessus, les membres de la famille d'hommes vivant avec un cancer de la prostate avancé AI connaissaient des problèmes et des besoins additionnels, sans doute dus à l'évolution de la maladie et aux symptômes associés.

\section{Le fardeau du soignant}

La plupart des proches hésitaient à discuter de leurs propres besoins et diminuaient l'importance de l'impact personnel de la vie quotidienne avec un homme atteint d'un CPA. Plusieurs proches du groupe AI ont décrit le fardeau associé au fait de satisfaire les besoins physiques, fonctionnels et affectifs des hommes. Une de ces femmes a indiqué que : "C'est un véritable combat de toujours tenter de lui montrer le côté positif. » Elle s'efforçait de projeter une attitude émotionnellement positive, ce qui lui était difficile. Une épouse du groupe AI a décrit le fardeau de fournir un soutien affectif à son mari sans dévoiler à leur famille et leurs amis l'ampleur de la maladie. Elle s'est exprimée ainsi : "Mais il a ce cancer depuis 1997, et vous ne pourriez jamais le deviner parce qu'il dit à tout le monde qu'il se porte à merveille. Lorsqu'on lui demande comment il va, sa réponse préférée est “Super bien!”. Je suis la seule à connaître la vérité. » Il lui était impossible d'obtenir du soutien pour elle-même sans trahir le souhait de son mari de garder le secret quant au cancer.

\section{Le besoin d'assistance pratique}

Les épouses des hommes atteints d'un cancer de la prostate avancé AI avaient besoin d'assistance pratique. Elles décrivaient leur incapacité à faire toutes leurs tâches ménagères. Elles devaient à présent faire des travaux que leur mari avait l'habitude de faire, comme l'entretien de la cour et le déblaiement de la neige. Elles devaient organiser les soins pour leur mari, y compris les tâches anodines comme la surveillance des médicaments. Très peu d'entre elles décrivaient à quel point ce travail additionnel était préjudiciable à leur santé, et certaines femmes mentionnaient être fatiguées. Une participante du groupe AI a décrit ainsi ses préoccupations : «Je ne veux pas me sentir indispensable, mais je me demande parfois ce qu'il ferait si je n'étais pas là, vous savez? Et je m'en fais pour ma propre santé. »

\section{L'isolement}

Pour certaines proches du groupe AI, le fardeau des soins menait à des sentiments d'isolement. Elles indiquaient que les hommes ne voulaient pas sortir en raison de l'incontinence et de la fatigue. Une fille adulte qui s'inquiétait de l'isolement de sa mère a affirmé que : "Ma mère a besoin de davantage d'activités. Elle a besoin de sortir et d'avoir davantage d'activités sociales, mais elle ne veut pas le quitter. Je crois qu'il aime voir de la famille, mais il veut rester à la maison. Elle ne veut pas nécessairement être sans cesse à la maison, et elle n'a pas toujours besoin d'y être non plus, donc nous sommes en présence de besoins complètement opposés en ce moment. » La fatigue constante d'un autre homme empêchait sa conjointe de recevoir de la visite à domicile. Les participantes avaient besoin de sortir et de rencontrer des gens, mais hésitaient à le faire. Une femme a indiqué que : «La situation affecte aussi ma vie sociale. Je ne me sens pas à l'aise de sortir et de m'amuser alors qu'il est coincé à la maison en train de souffrir. Parfois, je sais que je dois sortir. Mais je sais qu'il en ferait autant pour moi si les rôles étaient renversés. »

\section{Stratégies pour améliorer l'offre de soins de soutien}

La plupart des proches étaient satisfaits des soins que recevait leur être cher atteint d'un CPA. Parmi leurs suggestions, elles mentionnaient l'amélioration de l'accès à l'information. Elles voulaient un accès accru à des fournisseurs de soins bien renseignés. Elles voulaient passer plus de temps avec des personnes avisées qui comprenaient la condition de leur mari. Elles recommandaient que l'on établisse des centres de ressources pour les familles. Les proches des groupes HS et AI suggéraient également de créer des groupes de soutien pour les proches. Bon nombre de femmes mettaient l'accent sur le besoin de soutien pour chaque stade particulier du cancer de leur être cher. Seule une des femmes a demandé du soutien pour elle-même à son domicile.

\section{Discussion}

Autant que nous sachions, cette étude est la première évaluation des besoins qui cerne et compare les besoins des membres de la famille d'hommes atteints d'un cancer de la prostate avancé HS et AI et leur demande leurs suggestions pour l'amélioration de la prestation des soins. Les deux groupes de proches ont indiqué que leur besoin prioritaire en matière de soins de soutien était un besoin d'information. Lorsque Resendes et McCorkle (2006) ont analysé la documentation sur les réponses des épouses d'hommes diagnostiqués d'un cancer de la prostate qui avaient subi une prostatectomie, elles ont constaté que le manque d'information constituait une source de détresse. Dans une étude antérieure, les hommes atteints d'un cancer de la prostate (dont 59 \% en étaient au 
stade avancé) et leurs partenaires qui avaient pris part à des groupes de discussion avaient également signalé un besoin criant d'information (Harden et coll., 2002).

Les proches ont exprimé le besoin de recevoir plus d'information sur les traitements et les effets secondaires, sur les services de soutien offerts et sur la trajectoire future du cancer. Ce besoin est conforme aux résultats d'autres études (Butler, Downe-Wamboldt, Marsh, Bell \& Jarvi, 2000; Davison et coll., 2002; Finney Ruttin, Squiers \& Treiman, 2006; Giarelli et coll., 2003). La plupart des proches ont indiqué que leur principale source d'information était les professionnels de la santé. Ce constat contredit quelque peu les résultats d'une étude effectuée auprès d'hommes canadiens et de leurs partenaires par Davison et coll. (2002), dans laquelle les partenaires soutenaient que leurs principales sources d'information (en ordre d'importance décroissant) étaient les amis ou membres de la famille, d'autres hommes ayant un cancer de la prostate, la documentation disponible dans les cabinets de médecins, les médecins et Internet. Peu de proches ayant pris part à notre étude avaient recours à Internet. La vaste quantité d'information détaillée accessible dans Internet était une source de confusion et d'anxiété pour les proches.

La recherche antérieure révèle un conflit entre la recherche d'information et l'évitement de cette dernière. Dans leur étude sur les comportements de recherche d'information parmi les partenaires d'hommes vivant avec un cancer de la prostate, Feltwell et Rees (2004) ont constaté que les besoins en matière d'information variaient d'une personne à l'autre. Tandis que certaines cherchaient à obtenir de vastes quantités d'information, d'autres évitaient d'obtenir de l'information. Wong et ses collègues (2002), dans leur enquête auprès de patients canadiens atteints d'un cancer avancé et de leurs soignants, ont découvert qu'Internet était la source d'information la moins populaire. Les études de Wong et de Davison ont toutes deux été publiées en 2002, et il se peut que les opinions par rapport à Internet aient changé à mesure que les gens se sont habitués à l'utiliser.

Dans notre étude, les facteurs mentionnés par les proches qui aggravaient le manque d'information en provenance des professionnels de la santé étaient les problèmes de perte d'audition, la difficulté à comprendre l'information médicale, l'accès insuffisant aux médecins et la quantité insuffisante de temps passé avec ces derniers. Fitch (2006) a cerné de nombreuses difficultés éprouvées par les personnes âgées qui souhaitaient recevoir de l'information sur le cancer, y compris l'ambiance frénétique des milieux de soins de santé, le débit de parole trop rapide, l'utilisation de la terminologie médicale et le volume excessif d'information.

Les participantes aux groupes HS et AI ont toutes indiqué qu'elles se sentaient incertaines quant à leur avenir et à celui de leurs êtres chers. Elles avaient besoin de plus d'information sur la prochaine étape de traitement ou de soins de santé et sur ses conséquences. Shaha et ses collègues (2008) sont parvenus à un constat semblable lors de leur analyse documentaire. Celle-ci visait à cerner les principaux facteurs d'incertitude exprimés par les patients vivant avec des cancers mammaires, prostatiques et colorectaux et elle a révélé trois sources d'incertitude : 1) les enjeux liés à l'information après le diagnostic; 2) les décisions relatives au traitement et 3) l'effet du cancer sur la vie des patients et de leur famille au long de la trajectoire du cancer. Il s'agit là de défis particulièrement difficiles dans le cas du cancer de la prostate en raison des nombreuses décisions que les couples doivent prendre relativement aux traitements. Une autre étude a également révélé que les patients atteints d'un cancer terminal et leur famille veulent de l'information générale sur l'expérience de vie d'autres patients, mais ne veulent pas de détails sur leur propre situation (Clayton, Butow, Arnold \& Tattersall, 2005).
Resendes et McCorkle (2006), dans leur analyse des réactions psychologiques des épouses d'hommes qui avaient subi une prostatectomie, ont noté que la peur de l'inconnu et de l'avenir constituait une importante source de détresse. Nos résultats de recherche confirment ce constat. Dans leur étude qualitative, Harden et ses collègues (2002) ont utilisé des groupes de discussion pour explorer les expériences de couples vivant avec le cancer de la prostate. Ils ont cerné un thème émergent d'incertitude persistante qui se caractérisait par des difficultés avec les choix et des sentiments d'être sur des «montagnes russes émotionnelles ». Pour leur part, Kershaw et ses collaborateurs (2008) signalent que les épouses trouvaient que la communication avec leur mari aidait à réduire l'incertitude.

Les proches du groupe AI, mais non ceux du groupe HS, ont vécu de l'isolement. Plusieurs aspects du cancer de la prostate contribuent à l'isolement, notamment les changements dans l'activité sociale des participantes attribuables à des problèmes d'ordre physique comme l'incontinence et la fatigue. Harden (2005) a exploré les enjeux propres à la maladie parmi les couples touchés par le cancer de la prostate et a trouvé que les changements dans les schémas normaux de l'activité sociale menaient à des sentiments de solitude chez les soignants. Les épouses signalaient recevoir bien moins de soutien social que les patients dans une étude de Northouse et ses collègues (2007). Pour leur part, Butow et ses collaborateurs (2007) ont observé que les sentiments d'isolement constituent l'une des principales raisons pour lesquelles les patients et les soignants se joignent à des groupes de soutien. Dans notre étude, plusieurs femmes avaient cessé leurs activités habituelles parce qu'elles ne voulaient pas laisser leur mari seul. Une fille décrivait avec émotion les besoins opposés de son père et de sa mère; cette dernière avait besoin d'un exutoire social pour se distraire de ses activités de soignante.

Boehmer et Clark (2001b) ont décrit la façon dont les partenaires s'isolent souvent l'un de l'autre en évitant de communiquer et de travailler ensemble sur leurs émotions. Ces chercheurs ont également constaté que la communication des hommes au sujet de leur cancer se limite souvent à leur épouse. Ce constat était également manifeste dans notre étude. Une participante a déclaré : « Je suis la seule qui connait l'ampleur de la situation. » Kornblith et ses collègues (1994), dans leur étude sur la qualité de vie d'hommes et de leurs épouses, ont observé que les problèmes urinaires étaient associés plus fortement à la détresse des épouses qu'à celle des patients. Cette équipe de recherche soutient que la détresse provient des contraintes que les problèmes urinaires imposent sur la vie sociale du couple et/ou les peurs de gêne sociale liée à l'incontinence.

Harden (2005) a noté que les soignantes ont de nombreuses responsabilités à la maison, auxquelles s'ajoutent celles qui découlent de la fatigue et de l'état de santé de leur partenaire. Pour les conjointes plus âgées, ces responsabilités additionnelles s'ajoutent à leurs propres problèmes de santé et accroissent leur besoin de soutien (Hawes et coll., 2006). Dans notre étude, les proches avaient du mal à parler de leurs propres besoins, particulièrement ceux qui étaient liés au fardeau du soignant.

Sinfeld et ses collègues (2009) signalent que les couples veulent plus d'information afin qu'ils puissent s'occuper d'eux-mêmes à domicile. Dans notre étude, les proches indiquaient devoir faire de grands efforts pour obtenir des renseignements sur les services offerts ou pour obtenir des soins de soutien. La recherche antérieure révèle que les soignants naturels ne sont généralement pas au courant des services de soutien disponibles même lorsque les professionnels de la santé leur ont donné des renseignements à ce sujet (Hardwick \& Lawson, 1995). Galbraith et ses collaborateurs (2008) recommandent que les infirmières ne se contentent pas seulement d'inclure activement les partenaires lorsqu'elles offrent 
de l'information mais encore qu'elles les fassent participer aux processus de soins et qu'elles sollicitent leurs idées afin qu'ils soient bien informés.

Le manque de coordination et la distribution géographique diffuse des services offerts constituent des limites du système de santé. Plusieurs participantes ont recommandé la création de centres de ressources pour les familles afin de réunir les services disponibles et de rehausser l'accès aux professionnels de la santé. Wong et ses collègues (2002), dans leur enquête auprès de 71 patients canadiens vivant avec un cancer avancé et de 73 soignants, ont observé que la plupart des participants préféraient recevoir de l'information dans un cadre personnalisé avec un seul professionnel de la santé. Dans notre étude, les participantes ont également suggéré de former des petits groupes de soutien qui leur permettraient à la fois de recevoir de l'information et d'interagir avec d'autres personnes vivant une situation similaire, une recommandation déjà proposée par Harden et ses collègues (2002).

Une des limites de la présente étude est l'incohérence dans les méthodes de collecte de données. La plupart des proches du groupe AI ont participé à des groupes de discussion tandis que les proches du groupe HS ont pris part à des entrevues en personne. La décision d'utiliser ces deux méthodes nous a permis de recueillir de l'information auprès de personnes qui n'auraient peut-être pas participé autrement. Par exemple, certaines épouses ne voulaient pas laisser leur conjoint seul pour participer à un groupe de discussion. Une autre limite de notre étude est que nous utilisons les données recueillies auprès des participantes, mais nous ne saisissons pas les besoins des non-participants dans l'étude. Le groupe de proches non participants pourrait comprendre des personnes qui vont bien et n'ont pas recours aux services de soutien. Mais, fait plus important, il existe sans doute un groupe de proches qui sont vulnérables et peuvent souffrir en raison de leurs propres problèmes de santé et du manque de soutien social. Nous devons découvrir de nouvelles façons de mobiliser les proches les plus vulnérables aux besoins les plus criants de patients ayant un cancer afin de répondre à leurs besoins. Une des forces de notre étude est la possibilité de tirer des leçons de l'expérience de toute une gamme de proches qui vivent avec des patients atteints d'un CPA. Cette force découle du fait que nous avons recruté nos participantes de plusieurs manières (centre régional de cancérologie, cabinets d'urologues locaux, publicité dans la collectivité).

\section{Conclusions}

Les membres de la famille des hommes atteints d'un CPA jouent un rôle vital dans les soins, et nous devons mettre en place les services de santé qui les appuieront dans leurs rôles de soignantes. Ce besoin est urgent pour les épouses âgées d'hommes ayant un cancer de la prostate AI. Les demandes en matière de soins et les sentiments d'isolement sont accablants dans ce groupe. Les femmes peuvent avoir besoin d'un soutien additionnel à domicile afin de faire face aux responsabilités ménagères accrues qu'elles prennent en charge en raison de la détérioration de la fonction physique de leur mari. Le besoin des hommes de maintenir leur capacité fonctionnelle et la «normalité » peut être une source de conflit pour les proches qui offrent des soins à leurs êtres chers. Dans cette étude, les proches ont exprimé de la frustration découlant du stoïcisme des hommes vis-à-vis de la fatigue et de la douleur. Les membres de la famille avaient également besoin de soutien affectif pour les aider à composer avec leur propre peur, leur incertitude et leurs émotions liées à la maladie de leur partenaire. La recherche future devrait se pencher sur l'impact des besoins affectifs non remplis et de la détresse des proches sur la santé des hommes vivant avec un CPA. Kim et ses collègues (2008) ont constaté que la détresse des femmes était un bon prédicteur de la santé physique des hommes, indépendamment du niveau de détresse des hommes, de leur âge ou du stade de leur cancer.

La phase terminale de la maladie limite l'aptitude des proches à quitter la maison et à laisser leur être cher seul. Les services de soutien comme le « soutien de répit » ou les services d'aide familiale peuvent réduire le fardeau qui pèse sur les soignantes «confinées chez elles ", mais notre étude a cerné divers enjeux qui limitaient l'accès à ces services. Certaines participantes manquaient d'information sur les services existants, et d'autres hésitaient à demander de l'aide. Si nous souhaitons favoriser l'utilisation des services actuellement offerts, nous devons effectuer plus de recherche afin de comprendre la réticence de certains proches et patients à demander de l'aide.

Les membres de la famille de patients atteints d'un cancer ont besoin de davantage d'information pour les aider à prodiguer des soins à leurs êtres chers tout en réussissant leur propre adaptation (Finney Ruttin et coll., 2006). Nous recommandons la création et l'évaluation de petits groupes de soutien entre pairs-axés sur chaque stade de la maladie-à l'intention des proches d'hommes vivant avec un cancer de la prostate HS ou AI. Ces groupes seraient dirigés par des professionnels de la santé. Les groupes de soutien ciblés se sont avérés efficaces pour soutenir les personnes dont le conjoint avait éprouvé un incident cardiaque aigu (Gerwick, 1999) ou un traumatisme médullaire (Sheija \& Manigandan, 2005). Les participantes à notre étude voulaient avoir davantage de temps et d'interactions avec des professionnels de la santé comprenant bien le CPA. Ce projet de recherche visait à faire participer les familles à l'évaluation des services actuels et à leur demander leurs idées pour la refonte des services de soutien. En explorant les besoins des familles et en leur demandant leurs suggestions par rapport à la prestation de tels soins, nous avons pu cerner les priorités de cette population cible. La participation des familles dans la création et l'évaluation de nouveaux services est essentielle pour assurer l'utilisation de tels services.

\section{Annexe A. Guide d'entrevue}

1. De quelle façon votre vie et celle du membre de votre famille ont-elles été affectées par son diagnostic de cancer de la prostate avancé?

2. Quels besoins, problèmes ou préoccupations avez-vous (votre proche et vous) éprouvés depuis son diagnostic de cancer de la prostate avancé?

3. Dans quelle mesure ces besoins, problèmes et préoccupations ont-ils été abordés ou résolus?

4. Quelles stratégies-utiles et inutiles-avez-vous (vous et votre proche) employées pour composer avec ces besoins, problèmes ou préoccupations?

5. En quoi le système de santé (médecins, infirmières, centres de cancérologie et autres services de santé ou organismes communautaires) vous a-t-il aidée (ou non) à aborder ces besoins, problèmes ou préoccupations?

6. En vous basant sur votre expérience, pouvez-vous suggérer des façons dont la prestation de services de santé pourrait être améliorée en vue de mieux répondre à ces besoins?

7. Selon vous, quels besoins sont prioritaires pour les futurs efforts visant à améliorer les soins offerts aux patients et à leurs proches touchés par le cancer de la prostate avancé? 


\section{Références}

Boehmer, U., \& Clark, J.A. (2001b). Communication about prostate cancer between men and their wives. Journal of Family Practice, 50, 226-231.

Bryant-Lukosius, D., Browne, G., DiCenso, A., Whelan, T., Gafni, A., Neville, A., \& Sathya, J. (2010). Évaluation de la qualité de vie liée à la santé et des problèmes de santé prioritaires chez les patients atteints du cancer de la prostate : stratégie de définition du rôle des infirmières en pratique avancée. Revue canadienne de soins infirmiers en oncologie, 20(1), 5-14.

Bryant-Lukosius, D., \& DiCenso, A. (2004). A framework for the introduction and evaluation of advanced practice nursing roles. Journal of Advanced Nursing, 48, 530-540.

Butler, L., Downe-Wamboldt, B., Marsh, S., Bell, D., \& Jarvi, K. (2000). Behind the scenes: Partners' perceptions of quality of life post radical prostatectomy. Urologic Nursing, 20, 254-258.

Butow, P.N., Kirsten, L.T., Ussher, J.M., Wain, G.V., Sandoval, M., Hobbs, K.M., et al. (2007). What is the ideal support group? Views of Australian people with cancer and their carers. PsychoOncology, 16, 1039-1045.

Carter, N., Bryant-Lukosius, D., DiCenso, A., Blythe, J., \& Neville, A. (in press). The supportive care needs of men with advanced prostate cancer. Oncology Nursing Forum.

Clayton, J.M., Butow, P.N., Arnold, R.M., \& Tattersall, M.H. (2005). Discussing life expectancy with terminally ill cancer patients and their carers: A qualitative study. Supportive Care in Cancer, 13, 733-742.

Couper, J., Bloch, S., Love, A., Macvean, M., Duchesne, G. M., \& Kissane, D. (2006). Psychosocial adjustment of female partners of men with prostate cancer: A review of the literature. PsychoOncology, 15, 937-953.

Crowe, H., \& Costello, A.J. (2003). Prostate cancer: Perspectives on quality of life and impact of treatment on patients and their partners. Urologic Nursing, 23, 279-282.

Davison, B.J., Gleave, M.E., Goldenberg, S.L., Degner, L.F., Hoffart, D., \& Berkowitz, J. (2002). Assessing information and decision preferences of men with prostate cancer and their partners. Cancer Nursing, 25, 42-49.

Feltwell, A.K., \& Rees, C.E. (2004). The information-seeking behaviours of partners of men with prostate cancer: A qualitative pilot study. Patient Education \& Counseling, 54, 179-185.

Finney Ruttin, L.J., Squiers, L., \& Treiman, K. (2006). Requests for information by family and friends of cancer patients calling the national cancer institute's cancer information service. PsychoOncology, 15, 664-672.

Fitch, M. (2006). Perspectives of older adults about the cancer information they receive. European Journal of Oncology Nursing, 10, 216-217.

Galbraith, M.E., Pedro, L.W., Jaffe, A.R., \& Allen, T.L. (2008). Describing health-related outcomes for couples experiencing prostate cancer: Differences and similarities. Oncology Nursing Forum, 35, 794-801.

Gerwick, M.A. (1999). Impact of a focus support group on spouse's coping and support of their mate's recovery from an acute cardiac event (Unpublished Doctor of Philosophy thesis) University of Pittsburgh, Pittsburgh, PA.

Giarelli, E., McCorkle, R., \& Monturo, C. (2003). Caring for a spouse after prostate surgery: The preparedness needs of wives. Journal of Family Nursing, 9, 453-485.

Harden, J. (2005). Developmental life stage and couples' experiences with prostate cancer: A review of the literature. Cancer Nursing, 28, 85-98.

Harden, J., Schafenacker, A., Northouse, L., Mood, D., Smith, D., Pienta, K., et al. (2002). Couples' experiences with prostate cancer: Focus group research. Oncology Nursing Forum, 29, 701-709.
Harden, J.L., Northouse, L.L., \& Mood, D.W. (2006). Qualitative analysis of couples' experience with prostate cancer by age cohort. Cancer Nursing, 29, 367-377.

Hardwick, C., \& Lawson, N. (1995). The information and learning needs of the caregiving family of the adult patient with cancer. European Journal of Cancer Care, 4, 118-121.

Hawes, S.M., Malcarne, V.L., Ko, C.M., Sadler, G.R., Banthia, R., Sherman, S.A., et al. (2006). Identifying problems faced by spouses and partners of patients with prostate cancer. Oncology Nursing Forum, 33, 807-814.

Kershaw, T.S., Mood, D.W., Newth, G., Ronis, D.L., Sanda, M.G., Vaishampayan, U., et al. (2008). Longitudinal analysis of a model to predict quality of life in prostate cancer patients and their spouses. Annals of Behavioural Medicine, 36, 117-128.

Kim, Y., Kashy, D.A., Wellisch, D.K., Spillers, R.L., Kaw, C.K., \& Smith, T.G. (2008). Quality of life of couples dealing with cancer: Dyadic and individual adjustment among breast and prostate cancer survivors and their spousal caregivers. Annals of Behavioural Medicine, 35, 230-238.

Kornblith, A.B., Herr, H.W., Ofman, U.S., Scher, H.I., \& Holland, J.H. (1994). Quality of life of patients with prostate cancer and their spouses. Cancer, 73, 2791-2802.

Lavery, J.F., \& Clarke, V.A. (1999). Prostate cancer: Patients' and spouses coping and marital adjustment. Psychology, Health \& Medicine, 4, 289-302.

Maliski, S.L., Heilemann, M.V., \& McCorkle, R. (2002). From "death sentence" to "good cancer": Couples' transformation of a prostate cancer diagnosis. Nursing Research, 51, 391-397.

Manne, S., Babb, J., Pinover, W., Horwitz, E., \& Ebbert, J. (2004). Psychoeducational group intervention for wives of men with prostate cancer. Psycho-Oncology, 13, 37-46.

McMurtry, C.T., \& McMurtry, J.M. (2003). Metastatic prostate cancer: Complications and treatment. Journal of the American Geriatrics Society, 51, 1136-1142.

Northouse, L.L., Mood, D.W., Montie, J.E., Sandler, H.M., Forman, J.D., Hussain, M., et al. (2007). Living with prostate cancer: Patients' and spouses' psychosocial status and quality of life. Journal of Clinical Oncology, 25, 4171-4177.

Rees, C.E., Sheard, C.E., \& Echlin, K. (2003). The relationship between the information-seeking behaviours and information needs of partners of men with prostate cancer: A pilot study. Patient Education \& Counseling, 49, 257-261.

Resendes, L.A., \& McCorkle, R. (2006). Spousal responses to prostate cancer: An integrative review. Cancer Investigation, 24, 192-198.

Sandelowski, M. (2000). Whatever happened to qualitative description? Research in Nursing \& Health, 23, 334-340.

Sanders, S., Pedro, L.W., Bantum, E.O., \& Galbraith, M.E. (2006). Couples surviving prostate cancer: Long-term intimacy needs and concerns following treatment. Clinical Journal of Oncology Nursing, 10, 503-508.

Shaha, M., Cox, C.L., Talman, K., \& Kelly, D. (2008). Uncertainty in breast, prostate and colorectal cancer: Implications for supportive care. Journal of Nursing Scholarship, 40, 60-67.

Sheija, A., \& Manigandan, C. (2005). Efficacy of support groups for spouses of patients with spinal cord injury and its impact on their quality of life. International Journal of Rehabilitation Research, 28, 379-383.

Sinfeld, P., Baker, R., Camosso-Sefinovic, J., Colman, A.M., Tarrant, C., Mellon, J.K., et al. (2009). Men's and carers' experiences of care for prostate cancer: A narrative review. Health Expectations, 12, 303-312.

Wong, R.K., Franssen, E., Szumacher, E., Connolly, R., Evans, M., Page, B., et al. (2002). What do patients living with advanced cancer and their carers want to know? A needs assessment. Supportive Care in Cancer, 10, 408-415. 